\title{
Dynamics of electroencephalogram entropy and pitfalls of scaling detection
}

\author{
M. Ignaccolo, ${ }^{1}$ M. Latka, ${ }^{2}$ W. Jernajczyk, ${ }^{3}$ P. Grigolini, ${ }^{4}$ and B. J. West ${ }^{1,5}$ \\ ${ }^{1}$ Physics Department, Duke University, Durham, North Carolina, 27709 USA \\ ${ }^{2}$ Institute of Biomedical Engineering, Wroclaw University of Technology, Wroclaw, Poland \\ ${ }^{3}$ Department of Clinical Neurophysiology, Institute of Psychiatry and Neurology, Warsaw, Poland \\ ${ }^{4}$ Center for Nonlinear Science, University of North Texas, Denton, Texas, 76203 USA \\ ${ }^{5}$ Information Science Directorate, Army Research Office, Research Triangle Park, North Carolina, 27709 USA
}

(Received 12 June 2008; revised manuscript received 11 December 2009; published 10 March 2010)

\begin{abstract}
In recent studies a number of research groups have determined that human electroencephalograms (EEG) have scaling properties. In particular, a crossover between two regions with different scaling exponents has been reported. Herein we study the time evolution of diffusion entropy to elucidate the scaling of EEG time series. For a cohort of 20 awake healthy volunteers with closed eyes, we find that the diffusion entropy of EEG increments (obtained from EEG waveforms by differencing) exhibits three features: short-time growth, an alpha wave related oscillation whose amplitude gradually decays in time, and asymptotic saturation which is achieved after approximately $1 \mathrm{~s}$. This analysis suggests a linear, stochastic Ornstein-Uhlenbeck Langevin equation with a quasiperiodic forcing (whose frequency and/or amplitude may vary in time) as the model for the underlying dynamics. This model captures the salient properties of EEG dynamics. In particular, both the experimental and simulated EEG time series exhibit short-time scaling which is broken by a strong periodic component, such as alpha waves. The saturation of EEG diffusion entropy precludes the existence of asymptotic scaling. We find that the crossover between two scaling regions seen in detrended fluctuation analysis (DFA) of EEG increments does not originate from the underlying dynamics but is merely an artifact of the algorithm. This artifact is rooted in the failure of the "trend plus signal" paradigm of DFA.
\end{abstract}

DOI: 10.1103/PhysRevE.81.031909

PACS number(s): 87.19.1e, 05.40.-a, 05.10.Gg

\section{INTRODUCTION}

The neurons of the human brain form the most complex dynamical network ever studied. It is therefore hardly surprising that this complexity is reflected in electroencephalograms (EEG). Like most other biological time series, the EEG exhibits stochastic properties. Even when a person is quietly resting with eyes closed her EEG is irregular. However, an EEG time series is not simply uncorrelated noise but contains structure, such as alpha, beta, gamma and delta wave packets [1]. Consequently, EEG waveforms are nonstationary and require special methods for their analysis. A number of research groups [2-6] have determined that EEG time series have scaling properties, with a second moment that increases as a nontrivial power law in time.

The prevalent method used to determine the power-law index and to take into account the issue of nonstationarity is detrended fluctuation analysis (DFA) [7]. DFA is intended to remove the nonstationary components of the time series, called trends, and to provide a measure of the standard deviation of the detrended fluctuations as a function of the data window length. In the original formulation of DFA, physiological time series are integrated (accumulated) prior to detrending and calculation of standard deviations. However, the scaling properties of EEG dynamics were quantified not only by application of the original DFA to EEG signals $[3,5]$ but also without data integration $[4,5]$. The latter approach is equivalent to application of DFA to the differenced EEG time series or in other words to EEG increments. In this paper we investigate the scaling properties of EEG increments. The scaling of EEG waveforms is the subject of the companion paper.
Hwa and Ferree [4], for example, using the DFA of EEG increments found that the standard deviation exhibits two distinct scaling regions. They identify alpha waves as the cause of the crossover effect. Robinson [8], on the other hand, in his analysis of EEG time series, pointed out the existence of scaling up to a point after which saturation in the standard deviation occurred. He attributed this saturation effect to the influence of DFA detrending on the EEG Fourier spectrum. Thus, there are fundamental differences in interpretation of statistical properties of EEG fluctuations.

Herein we demonstrate that the Shannon entropy implemented using the diffusion entropy approach [9] provides insight into the EEG dynamics. The functional form of the time evolution of diffusion entropy suggests a linear Ornstein-Uhlenbeck Langevin equation (OU Langevin equation) as a suitable mathematical framework [10]. The statistical properties of the OU Langevin model can be determined analytically and this is why it is the starting point of our analysis of scaling in EEG time series.

In Sec. II we introduce stochastic differential equations (Langevin equations) as a way to model complex phenomena. Using diffusion entropy analysis (DEA) we show how the EEG dynamics of subjects in the resting condition can be described by an Ornstein-Uhlenbeck Langevin equation with a quasiperiodic forcing that mimics the dynamics of alpha waves. The coherence of the periodic modulation is also discussed. In Sec. III we introduce the DFA, an increasingly popular technique in the neuroscience literature for the analysis of EEG time series. We then compare the results of the DFA and DEA of (1) the numerical solution of the OU Langevin equation, (2) the numerical solution of the $\mathrm{OU}$ Langevin equation with the quasiperiodic forcing, and (3) the EEG records. We discuss the influence of alpha waves on the 
outcome of DEA and DFA. In Sec. IV the inherent limitations of DFA of EEG time series are discussed. We present our conclusions in Sec. V.

\section{EEG MODELS}

In this Section, we first introduce the Ornstein-Uhlenbeck Langevin equation, which is the simplest dynamical model including both fluctuation and dissipation (Sec. II A). We then review the DEA and calculate the information entropy for the OU Langevin equation (Sec. II B). In Sec. II C we identify the distinct features of the time evolution of diffusion entropy of EEG increments. Finally, we extend the OU Langevin model by adding a quasiperiodic forcing to mimic the dynamics of alpha waves which are usually strongly pronounced in awake subjects with closed eyes (Sec. II D).

\section{A. Ornstein-Uhlenbeck Langevin equation}

In the physics literature there are two main strategies for treating stochastic phenomena (see for example, Lindenberg and West [11]). The first employs stochastic differential equations, Langevin equations, to model the evolution of dynamical variables. In the second strategy the time evolution of the probability density in phase space is determined using partial differential equations known as Fokker-Planck equations. In this work we choose a Langevin equation to model the EEG dynamics.

Let us recall that the OU Langevin equation for a linearly dissipative stochastic process reads

$$
\frac{d X(t)}{d t}=-\lambda X(t)+\eta(t)
$$

where $\lambda$ is the dissipation rate and the Gaussian random force $\eta(t)$ is delta correlated in time with strength $D$

$$
\langle\eta(t) \eta(t+\tau)\rangle=2 D \delta(\tau)=\sigma_{\eta}^{2} \delta(\tau) .
$$

In the above equation the symbol $\langle\ldots\rangle$ indicates the Gibbsean ensemble average and $\sigma_{\eta}$ is the standard deviation of the noise $\eta$.

In the context of physiological time series analysis, we refer to an average $\langle\ldots\rangle$ over statistically independent realizations of random walk Eq. (1) as a multiple trajectory ensemble (MTE) average. However, very seldom do physiological data allow for such averaging. In most applications expectation values must be calculated by partitioning a single experimental time series. For brevity, we call such an approach the single trajectory ensemble (STE) averaging.

Equation (1) contains two physical mechanisms; the influence of the environment, which gives rise to the random force, and the dissipation, which models the average energy extracted from the dynamic system and absorbed by the environment.

The solution of Eq. (1) is

$$
X(t)=e^{-\lambda t} \int_{0}^{t} \eta\left(t^{\prime}\right) e^{\lambda t^{\prime}} d t^{\prime},
$$

where we assumed without loss of generality that $X(0)=0$. Using Eq. (3) it is possible to calculate the variance of $X(t)$

$$
\sigma^{2}(t) \equiv\left\langle X^{2}(t)\right\rangle-\langle X(t)\rangle^{2}=\frac{D}{\lambda}\left[1-e^{-2 \lambda t}\right] .
$$

Consequently, for $t \ll 1 / \lambda$ the variance increases linearly with time

$$
\lim _{t \rightarrow 0} \sigma^{2}(t) \approx 2 D t
$$

It follows from the above equation that in the short-time limit

$$
\log _{2} \sigma(t) \approx \frac{1}{2} \log _{2} t+\text { const. }
$$

Thus, the short-time scaling index for the OU Langevin equation is equal to 0.5 . See $[12,13]$ for a review of scaling in the natural sciences and medicine.

For $t \gg 1 / \lambda$ the variance becomes time independent

$$
\lim _{t \rightarrow \infty} \sigma^{2}(t)=\frac{D}{\lambda}
$$

with a saturation induced by the dissipation. This saturation precludes the asymptotic scaling of the OU Langevin model.

\section{B. Diffusion entropy analysis}

The solution to the OU Langevin equation, Eq. (3), defines a stochastic trajectory whose statistical properties are described by the probability density function (pdf) $p(x, t)$ : the probability density of finding the trajectory in an infinitesimal neighborhood of $x$ at time $t$. The pdf is determined from the histogram of $X(t)$ and may be used to calculate the information entropy. This concept was introduced in discrete form for coding information by Shannon [14] and is now commonly referred to as the Shannon entropy. The continuous form

$$
S(t)=-\int p(x, t) \log _{2} p(x, t) d x
$$

was first applied by Wiener to the problem of noise and messages in electrical filters [15]. In the context of time series analysis $S(t)$ was named diffusion entropy [9].

Entropy is frequently used to quantify variability of time series. One of the advantages of this measure over the variance is that the entropy gives a more complete description of the stochastic process when the pdf is not Gaussian, e.g., the EEG records studied here. If the pdf scales in the following way:

$$
p(x, t)=\frac{1}{\beta(t)} F\left(\frac{x}{\beta(t)}\right)
$$

then it is straightforward to show that the entropy reads

$$
S(t)=-\int F(y) \log _{2} F(y) d y+\log _{2} \beta(t) .
$$

As a consequence, when the scaling function is a power law in time 


$$
\beta(t) \propto t^{\delta} \Rightarrow S(t)=\delta \log _{2} t+\text { const }
$$

the diffusion entropy increases linearly on a log-linear plot, with the slope equal to the scaling exponent $\delta$. Note that the scaling of the pdf given by Eq. (9) is satisfied by a Gaussian process, with $\beta(t)$ being the time-dependent standard deviation $\sigma(t)$. Even when the distribution is Gaussian, there is no guarantee that the standard deviation increases as a power law in time as, for example, in Eq. (5). Finally, processes such as Lèvy flights may satisfy the scaling condition for the pdf but do not have a finite variance. In this case $\beta(t)=t^{1 / \alpha}$, where $\alpha$ is the Lèvy index. It is worth emphasizing that for Lèvy flights $\beta^{2}$ is not the variance of fluctuations. Consequently, any second moment analysis is useless in determining the scaling exponent $1 / \alpha$, while DEA does yield the correct estimate. Also in the case of Lèvy walks, which do have finite variance, DEA correctly detects the Lèvy scaling of the central part of the pdf unlike second moment methods [16].

The applicability of DEA goes far beyond scaling detection. For example, this algorithm has been successfully used to determine the seasonal influence and the demographic pressure on the daily number of teen births in Texas [17], to study the effect of solar cycles on the statistics of solar flares [18], and to investigate the influence of solar dynamics on the fluctuations of average global temperature [19]. Recently, the DEA has been used to characterize statistical properties of the EEG time series [10].

We now calculate the information entropy $S(t)$ for the $\mathrm{OU}$ Langevin equation (1). Given the Gaussian statistics of the random force and the linear form of the equation we know that the fluctuations of the dynamical variable $X(t)$ are also Gaussian. Substituting a Gaussian distribution with a variance $\sigma^{2}(t)$ into Eq. (8) we obtain

$$
S(t)=\log _{2}[\sqrt{2 \pi e} \sigma(t)]=\frac{1}{2} \log _{2}\left[\frac{2 \pi e D}{\lambda}\left(1-e^{-2 \lambda t}\right)\right] .
$$

Consequently, employing the approximate variance Eq. (5) we can show that for $t \ll 1 / \lambda$ the entropy increases as

$$
\lim _{t \rightarrow 0} S(t)=\frac{1}{2} \log _{2}(4 \pi D e)+\frac{1}{2} \log _{2} t
$$

and a linear-log plot yields a straight line of slope $1 / 2$, the value of the short-time scaling index Eq. (6). At the other extreme $t \gg 1 / \lambda$, using the approximate variance Eq. (7) we can calculate the saturation level of the diffusion entropy $S_{\infty}$,

$$
S_{\infty}=\lim _{t \rightarrow \infty} S(t)=\frac{1}{2} \log _{2}\left(\frac{2 \pi D e}{\lambda}\right) .
$$

\section{Diffusion entropy of EEG waveforms}

Let us consider a time series $\left\{\xi_{j}\right\}_{j=1}^{N}$ of length $N$ of an evenly sampled waveform. In order to estimate the entropy, Eq. (8), of this data segment we first construct a set of $M$ $=N-t+1$ diffusive trajectories,

$$
Z_{k}(t)=\sum_{j=k}^{k+t-1} \xi_{j} \quad k=1,2, . ., N-t+1 .
$$

This procedure amounts to accumulating all possible $t$ consecutive data points $\xi_{j}$. The trajectories $Z_{k}(t)$ are used to evaluate the pdf $p(Z=x, t)$, the probability density for the variable $Z$ to be in an infinitesimal neighborhood of $x$ at time $t$. For each value of the variable $t$ a bin size $\Delta(t)$ is selected and the histogram of the number of points falling in each bin is calculated. The bin size $\Delta(t)$ is chosen as a predetermined fraction of the standard deviation of the sequence $\left\{Z_{k}(t)\right\}$. Then the summation over bins in Eq. (8) is performed to estimate the information entropy $S(t)$. The adoption of the time-dependent bin size prevents a lack of statistics due to the spreading of the trajectories $Z_{k}(t)$ with time. In other words, if the bin width was fixed, the number of points falling in some bins could become very low with the passage of time. A detailed description of the numerical implementation of the DE method can be found in [17].

Here, $\left\{\xi_{j}\right\}_{j=1}^{N}$ is the time series of the EEG increments obtained from EEG waveforms by ordinary differencing. We refer to the corresponding information entropy as the EEG entropy. The data set for the analysis was comprised of EEG records of twenty awake healthy volunteers. EEG monitoring was performed for subjects in a supine position, in the absence of external stimulation and with eyes closed. The EEG was recorded using the 10-20 standard of electrode placement with a sampling frequency of $250 \mathrm{~Hz}$. For each subject we selected an artifact free EEG epoch (segment). The length of these segments varied from 55 to $400 \mathrm{~s}$ with mean value of $128.1 \mathrm{~s}$. For eight individuals only the channels O1, O2, C3, and C4 were recorded, for the remaining twelve all 19 leads were used. Consequently, the statistical analysis was restricted to the channels $\mathrm{O} 1, \mathrm{O} 2, \mathrm{C} 3$, and $\mathrm{C} 4$, which are the channels traditionally used in sleep studies. The absolute amplitude of EEG is irrelevant both in entropy calculations and scaling analysis. Therefore we expressed the amplitudes of EEG waveforms in the units of analog-to-digital converter (559 units correspond to $10 \mu \mathrm{V}$ ).

Figure 1 shows the diffusion entropy for channel $\mathrm{O} 1$ of one of the subjects. We can see in this figure three features which turn out to be generic: (1) initial fast growth of entropy, (2) "alpha" ( $7.6 \mathrm{HZ}$ in the case of this subject) modulation which is attenuated with time, and (3) asymptotic saturation. As expected, the modulation is strongly pronounced in the occipital region where the amplitude of alpha waves is high. This effect is illustrated by Fig. 2 where the time evolution of diffusion entropy is plotted for all 19 channels of one of the subjects.

The inset in Fig. 1 depicts the pdf $p_{\text {sat }}(x)$ (solid line) after entropy saturation is attained. This distribution is not Gaussian as indicated by the deviation of the best Gaussian fit (solid thick line) from the tails of $p_{\text {sat }}(x)$. These tails can be well approximated by an exponential function: $C \exp (-\theta x)$. The asymptotic probability density function in this figure is typical of the cohort of subjects.

\section{EEG OU Langevin equation}

To account for all three features of EEG diffusion entropy (initial growth, possible alpha wave modulation and 


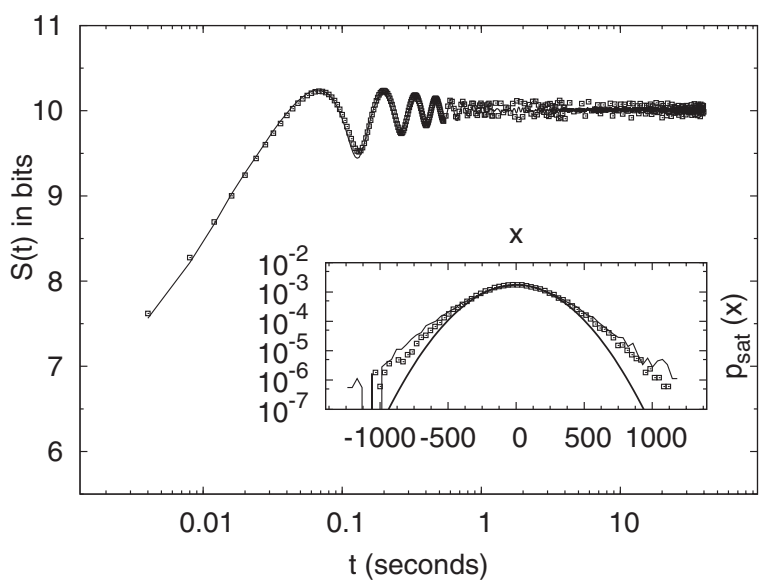

FIG. 1. Time evolution of diffusion entropy for: channel O1 (solid line) and the increments of the variable $X$ which is the solution of the EEG OU Langevin equation (16) (squares). Inset: the comparison between the asymptotic probability density functions at $t=8 \mathrm{~s}$ for: channel $\mathrm{O} 1$ (solid line), variable $X$ of Eq. (16) (squares), and the best Gaussian fit to the pdf of the experimental data (thick line).

asymptotic saturation) we extend the OU Langevin model equation (1) by adding quasiperiodic driving with random amplitude and frequency

$$
\frac{d X(t)}{d t}=-\lambda X(t)+\eta(t)+\sum_{j=0} A_{j} \chi_{j, s}(t) \sin \left[2 \pi f_{j} t\right] .
$$

For brevity, we dub this equation the EEG OU Langevin equation. The amplitude $A_{j}$ and the frequency $f_{j}$ of the driving remain constant in the time interval $\left[j t_{s},(j+1) t_{s}\right]$, $(j=0, \ldots, N-1)$, of duration $t_{s}$. The function $\chi_{j, s}$ is defined as

$$
\left\{\begin{array}{ll}
\chi_{j, s}(t)=1 & \text { if } t \in\left[j t_{s},(j+1) t_{s}\right] \\
\chi_{j, s}(t)=0 & \text { otherwise }
\end{array},\right.
$$

$t_{s}$ is the "stability" time after which a new frequency and amplitude are selected. The parameters of the driving are determined from the EEG spectrogram. For each time interval $j$ of duration $t_{s}$ we search for the peak in the alpha wave

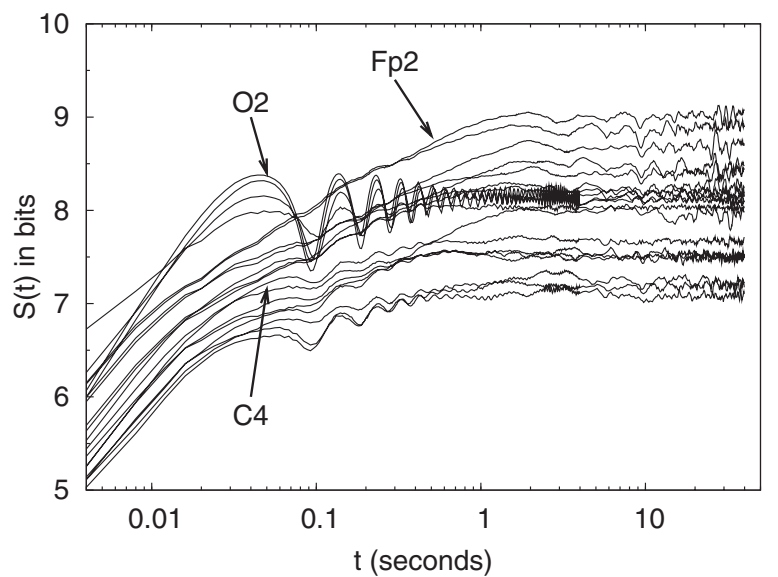

FIG. 2. Time evolution of diffusion entropy $S(t)$ of all 19 EEG channels of one subject. spectrum $(7-12 \mathrm{~Hz})$. The location and the amplitude of this peak determine $f_{j}$ and $A_{j}$. We arbitrarily set the stability time $t_{s}$ to $0.5 \mathrm{~s}$. This choice provides a reasonable tradeoff between time and frequency resolution. The influence of the stability time on the dynamics of the EEG OU Langevin equation is analyzed in Appendix. A detailed description of the numerical algorithm may be found in [10].

Assuming, as before, without loss of generality that $X(0)=0$, the formal solution to Eq. (16) reads

$$
X(t)=e^{-\lambda t} \int_{0}^{t}\left\{\eta\left(t^{\prime}\right)+\sum_{j=0} A_{j} \chi_{j, s}(t) \sin \left(2 \pi f_{j} t^{\prime}\right)\right\} e^{\lambda t^{\prime}} d t^{\prime} .
$$

Averaging over the MTE, we may obtain an expression for the standard deviation of the variable $X$,

$$
\begin{aligned}
\sigma^{2}(t)= & \frac{D}{\lambda}\left[1-e^{-2 \lambda t}\right]+\frac{e^{-2 \lambda t}}{4}\left[\sum _ { j = 0 } A _ { j } \int _ { 0 } ^ { t } \chi _ { j , s } ( t ) \left\{e^{(\lambda+2 \pi i f) t^{\prime}}\right.\right. \\
& \left.\left.+e^{(\lambda-2 \pi i f) t^{\prime}}\right\} d t^{\prime}\right]^{2}
\end{aligned}
$$

If we consider a monochromatic alpha wave $\left(\forall j A_{j}=A\right.$ and $f_{j}=f$ ) then Eq. (16) describes a stationary ergodic process. In this case Eq. (19) reduces to

$$
\begin{aligned}
\sigma^{2}(t)= & \frac{D}{\lambda}\left[1-e^{-2 \lambda t}\right]+\frac{A^{2} e^{-2 \lambda t}}{\left(\lambda^{2}+4 \pi^{2} f^{2}\right)^{2}} \\
& \times\left[e^{\lambda t} \sqrt{\lambda^{2}+4 \pi^{2} f^{2}} \cos (2 \pi f t+\phi)+2 \pi f\right]^{2}
\end{aligned}
$$

with $\phi=\arctan (\lambda / 2 \pi f)$. In contrast with the standard OU Langevin equation [cf. Eq. (5)], the variance in Eq. (20) does not exhibit algebraic scaling for $t \ll 1 / \lambda$. For the asymptotic behavior $(t \rightarrow \infty)$ we get

$$
\lim _{t \rightarrow \infty} \sigma^{2}(t)=\frac{D}{\lambda}+\frac{A^{2}}{\lambda^{2}+4 \pi^{2} f^{2}} \cos (2 \pi f t+\phi) .
$$

Thus, the asymptotic variance oscillates around the mean value $D / \lambda$.

Periodic driving (monochromatic or not) induces a departure from the Gaussian distribution of $X$. The inset in Fig. 1 illustrates this effect. As a consequence, the $S(t)$ and $\sigma(t)$ are no longer related through Eq. (12), although the standard deviation can still be considered as a proxy measure of the entropy: larger standard deviation implies larger entropy.

In the case of a nonmonochromatic alpha waves the stochastic process described by Eq. (16) is nonstationary and thus nonergodic. The MTE average reads

$$
\sigma^{2}(t)=\frac{D}{\lambda}\left[1-e^{-2 \lambda t}\right]+e^{-2 \lambda t}\left\{\sum_{j=0}^{j_{0}-1} \Pi_{j}\left[(j+1) t_{s}\right]+\Pi_{j_{0}}(t)\right\}^{2}
$$

where $j_{0}$ is such that $j_{0} t_{s}<t<\left(j_{0}+1\right) t_{s}$, 
TABLE I. The group-average values of the parameters $\lambda$ and $\sigma_{\eta}$ of Eq. (16).

\begin{tabular}{lcc}
\hline \hline EEG channel & $\lambda($ mean \pm std $)$ & $\sigma_{\eta}($ mean \pm std $)$ \\
\hline O1 & $0.05 \pm 0.02$ & $16 \pm 7$ \\
O2 & $0.05 \pm 0.02$ & $16 \pm 7$ \\
C3 & $0.04 \pm 0.02$ & $10 \pm 4$ \\
C4 & $0.04 \pm 0.02$ & $11 \pm 4$ \\
\hline \hline
\end{tabular}

$$
\begin{aligned}
\Pi_{j}(t)= & \frac{A_{j}}{\left(\lambda^{2}+4 \pi^{2} f_{j}^{2}\right)}\left\{\sqrt { \lambda ^ { 2 } + 4 \pi ^ { 2 } f _ { j } ^ { 2 } } \left[e^{\lambda t} \cos \left(2 \pi f_{j} t+\phi_{j}\right)\right.\right. \\
& \left.\left.-e^{\lambda j t_{s}} \cos \left(2 \pi f_{j} j t_{s}+\phi_{j}\right)\right]\right\},
\end{aligned}
$$

and $\phi_{j}=\arctan \left(\lambda / 2 \pi f_{j}\right)$. Time dependence of the variance in Eq. (22) is similar to that of Eq. (20). In fact, the coefficient $e^{-2 \lambda t}$ attenuates all modulation terms except $\Pi_{j_{0}}^{2}(t)$. Considering only the nonattenuated contribution, we have the same behavior as in Eq. (20) and asymptotically-Eq. (21). The overall effect of the attenuated terms amounts to the modification of the mean value around which the oscillations occur. This similarity to the monochromatic case is due to the fact that at any time $t\left(j t_{s}<t<(j+1) t_{s}\right)$ all the trajectories of the MSE have the same amplitude and frequency for the alpha wave modulation: $A_{j}$ and $f_{j}$. However, this condition is broken when time averages on a single trajectory are performed. In the latter case we are forced to use fixed length portions of the trajectory as different samples of the ensemble. Thus, for a fixed $t$ the amplitude and frequency of the alpha modulation may vary in the ensemble which results in decaying oscillation exhibited by both the variance and the information entropy. In Fig. 1, one can see these damped oscillations of the diffusion entropy for the EEG (solid line) and simulated EEG (squares). The agreement of these two curves is excellent. The agreement extends also to the pdfs after saturation $p_{\text {sat }}(x)$ : compare the thin solid line to the squares in inset of Fig. 1.

In Table I the group-average values of the best-fit parameters $\lambda$ and $\sigma_{\eta}$ are presented as mean \pm standard deviation (std) for channels C3, C4, O1, and O2. For each data segment of a given volunteer these two parameters were independently varied to achieve the best possible agreement between the time evolution of diffusion entropy of EEG and that of the model Eq. (16). The amplitudes and the frequencies of the quasiperiodic driving remained constant during the fitting procedure. Note that the occipital channels are generally noisier than the central channels.

\section{DEA VERSUS DFA}

In this section, we first review detrended fluctuation analysis (Sec. III A) which in neuroscience is the most commonly used scaling detection algorithm. Then, in Sec. III B, both DFA and DEA are applied to: the numerical solution of the OU Langevin equation (1), the numerical solution of the EEG OU Langevin model equation (16), and EEG waveforms. We carry out calculations using the increments of ei- ther the stochastic variable $X(t)$ or EEG. The rationale for the comparison of DFA to DEA was provided by the theoretical analysis of the dynamics of the EEG OU Langevin model. In particular, it is apparent from Eqs. (20) and (22) that in the presence of strong monochromatic or quasimonochromatic driving the variance of the dynamical variable $X(t)$ does not exhibit short-time scaling $(t \ll 1 / \lambda)$. Thus, the question arises as to the influence of alpha waves on the outcome of scaling analysis of EEG. This question is particularly pertinent to DFA, which employs polynomial detrending. Such detrending implies that EEG is made up of trends, to a large extent perceived as unwanted "noise," superposed on the actual signal whose statistical properties are the subject of analysis. We focus attention on the validity of such decomposition.

\section{A. Detrended fluctuation analysis}

We now briefly describe the DFA algorithm [7]. Given a time series $\left\{\xi_{j}\right\}_{j=1}^{N}$, the zero-centered time series is aggregated

$$
Z_{k}=\sum_{j=1}^{k}\left[\xi_{j}-\xi_{a v g}\right] \quad k=1,2, \ldots, N,
$$

where $\xi_{a v g}=(1 / N) \sum_{j=1}^{N} \xi_{j}$. The integrated signal $\left\{Z_{k}\right\}$ is divided into $n_{t}$ nonoverlapping windows of size $t\left(n_{t}=N / t\right)$. For each window $l=1, . ., n_{t}$, a least-squares polynomial fit $y_{l}^{(m)}(k)$ is computed. The degree of the polynomial $m$ can be varied in order to eliminate linear $(m=1)$, quadratic $(m=2)$ or higher order trends. The fitted trend is subtracted from the integrated time series and the standard deviation $F_{l}(t)$ of residuals $\widetilde{Z}_{k, l}=Z_{k, l}-y_{l}^{(m)}(k)$ is calculated,

$$
F_{l}(t)=\sqrt{\frac{1}{t} \sum_{k=1}^{t} \tilde{Z}_{k, l}^{2}(t) .}
$$

After averaging $F_{l}$ over all $n_{t}$ windows we obtain the mean value $F(t)$. These steps are repeated for increasing values of the window size $t$. The scaling condition for the standard deviation $F(t) \propto t^{\alpha}$ implies

$$
\log _{2} F(t) \propto \delta \log _{2} t .
$$

DFA may be performed for either nonoverlapping or sliding windows. The latter variant is available in the original implementation of DFA available at the Physionet website (http:// www.physionet.org [20]).

The DFA assumes [21] the time series $\left\{Z_{k}\right\}$ to be the superposition of two independent contributions: the trend $T$ and the signal $S$

$$
Z_{k}=T_{k}+S_{k}
$$

with the zero covariance $[\operatorname{Cov}(T, S)=0]$. However, the outcome of DFA does not by itself allow for the validation of the decomposition assumption Eq. (27). As we will show, DFA must not be indiscriminately applied, since there are many dynamical systems for which Eq. (27) is unphysical. Linear dependence of $\log _{2} F(t)$ on $\log _{2} t$ does not necessarily imply the existence of underlying power-law correlations but may be just an artifact of data analysis. The difficulties of scaling analysis of physiological time series are compounded 


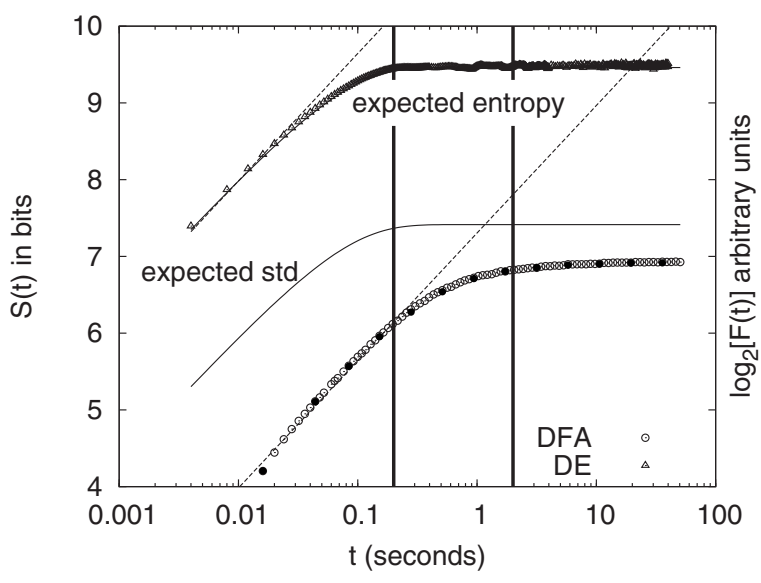

FIG. 3. DEA and DFA of the dynamics of the stochastic variable $X(t)$ which is the solution of the OU Langevin equation Eq. (1) with $\lambda=0.055$ and $\sigma_{\eta}=40$. The triangles denote the values of the diffusion entropy $S(t)$ while open circles represent the values of base- 2 logarithm of $F(t)$. The filled circles correspond to the DFA calculations with nonoverlapping windows. The solid lines indicate the analytical values for $S(t)$ and standard deviation of $X(t)$, cf. Eqs. (12) and (4), respectively. Note that these analytical values were calculated for the effective dissipation rate $\lambda_{e f f}=\lambda / 2$, see the discussion in the text. The dashed lines were drawn to guide the eye. Their slopes are equal to 0.5 which is the value of short-time scaling exponent for the OU Langevin equation (1).

by the fact that quite often one deals not only with short-time scaling but also with crossover effects (different scaling regions).

In the next subsection we carry out the scaling analysis using both DFA and DEA. To make a more direct comparison we employ the overlapping window variant of DFA. However, the original nonoverlapping algorithm did not yield qualitatively different results. We have chosen a linear detrending.

\section{B. Comparison of DEA and DFA}

Figure 3 illustrates DEA and DFA of the increments of the variable $X(t)$. The initial value problem for $X(t)$ was solved by the numerical integration of Eq. (1) with $\lambda=0.055$ and $\sigma_{\eta}=40$. Please note that in Sec. II we derived the analytical formulas for the diffusion entropy and standard deviation of the OU Langevin equation, cf. Eqs. (12) and (4), respectively. However, these expressions are not directly applicable to DEA or DFA. Recall that they were derived using the ensemble of statistically independent trajectories originating at $X=0$. However, both in DEA and DFA averaging involves relative displacements $Z_{k}(t)$,

$$
Z_{k}(t)=X(t+k)-X(k) \quad k \in[0, N-t],
$$

cf. Eq. (15). Consequently, it can be shown that the expressions for the standard deviation Eq. (4) and diffusion entropy Eq. (12) of the OU Langevin equation must be modified. Calculations performed in [22] show that required modification is surprisingly simple and amounts to replacing the dissipation rate in Eqs. (4) and (12) by the effective dissipation rate,

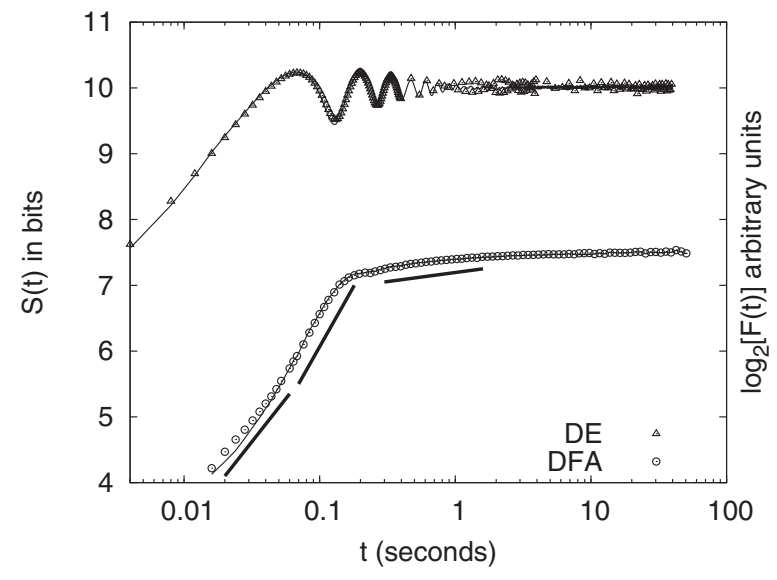

FIG. 4. DEA and DFA of the EEG segment extracted from channel $\mathrm{O} 1$ of one of the subjects (solid lines). Both analyses were also performed for the stochastic variable $X(t)$ - the solution of the EEG OU Langevin equation (16) with $\lambda=0.055$ and $\sigma_{\eta}=40$. Triangles and circles in this plot correspond to the diffusion entropy $S(t)$ and standard deviation $F(t)$ of the DFA residuals of the simulated EEG, respectively. The thick solid lines indicate three pseudoscaling regions.

$$
\lambda_{e f f}=\lambda / 2 \text {. }
$$

One can see in Fig. 3 that the time evolution of diffusion entropy (triangles) closely follows the theoretical prediction given by Eq. (12) with the modified dissipation rate $\lambda_{e f f}$. In particular, the slope of the initial linear growth of diffusion entropy in this semilog plot coincides with the analytical value 0.5 [Eq. (13)]. The values of $F(t)$ (open circles) are significantly smaller than those given by Eq. (4) with the modified dissipation rate $\lambda_{e f f}$. This is not surprising taking into account that $F(t)$ is the standard deviation of detrended fluctuations. However, we can see in Fig. 3 that $F(t)$ approaches the asymptotic value at least a decade later than does the corresponding theoretical curve. We elucidate the origin of this discrepancy in Sec. IV. For the arbitrary choice of $0.04 \mathrm{~s} \leq t \leq 0.4 \mathrm{~s}$ we obtain the slope of 0.44 , slightly smaller than the analytical value of 0.5 .

The filled circles in Fig. 3 denote $F(t)$ for the nonoverlapping window variant of DFA. One can see that the agreement with the sliding-window calculations is good.

In Fig. 4 the diffusion entropy of the EEG segment extracted from the channel $\mathrm{O} 1$ of one of the volunteers is plotted as a function of time (solid line in the upper half of the graph). The triangles denote the diffusion entropy of the simulated EEG, generated by integration of the EEG OU Langevin equation $\left(\lambda=0.055\right.$ and $\left.\sigma_{\eta}=40\right)$. The growth of the diffusion entropy of the EEG waveform (in the semilog plot) is essentially linear with the slope $1.07 \quad(0.004 \mathrm{~s}<t$ $<0.04 \mathrm{~s})$. Note that the short-time scaling exponent for the simple OU Langevin equation is 0.5 , which is just the manifestation of a lack of correlations for the stochastic variable $\eta[$ cf. Eq. (1)]. Thus, taking into account the agreement between the diffusion entropy of the experimental data and that of the EEG OU Langevin model equation (16) in Fig. 4, the observed difference in scaling exponents must be caused by the strong alpha waves in the occipital channel. It turns out 
that for times smaller than the typical period of the alpha waves, the dynamical effect of these oscillations amount to trends which enhance the initial growth of entropy, cf. the short-time limit of Eq. (22). Consequently, in this case, the value of the scaling exponent is not a straightforward indicator of correlations in EEG time series. For a detailed discussion of the influence of periodic driving on the dynamics of diffusion entropy see [17].

One might hope that polynomial detrending of DFA could alleviate the difficulties of EEG scaling analysis. In fact, in Fig. 4 the standard deviation $F(t)$ does not exhibit the alpha wave modulation characteristic of diffusion entropy. However, one can identify two pseudoscaling regions with different slopes: 0.65 for $0.01 \mathrm{~s}<t<0.06 \mathrm{~s}$ and 1.2 for $0.08 \mathrm{~s}$ $<t<0.2$ s. None of these slopes, represented in Fig. 4 by thick solid lines, correspond to genuine dynamical scaling.

Using the analytical formula for the standard deviation for the OU Langevin equation, we have identified the limitation of DFA: the slow approach of $F(t)$ to its asymptotic value (Fig. 3). The same effect may be observed in Fig. 4 for both the EEG waveform and the simulated time series. One can see that $F(t)$ continues to grow long after the diffusion entropy $S(t)$ reaches the saturation level. Such spurious, slow growth may be easily misinterpreted as yet another scaling region. The third thick line in Fig. 4 represents the slope of the linear least-squares fit to $F(t)$ of EEG for $0.3 \mathrm{~s}<t$ $<1.6$ s. Such an interval was used by Hwa and Ferree in their studies of the scaling properties of EEG [4]. However, such an interval lies within the saturation region so that the justification of scaling analysis is lost.

\section{DISCUSSION}

Let us analyze the results of the previous studies of the scaling properties of EEG increments from the perspective of the OU Langevin equation (1) and its extension Eq. (16). As we demonstrated, these models capture the salient features of EEG dynamics.

Hwa and Ferree [4] argue that the fluctuations of short $(\sim 10$ s) EEG segments may be quantified by two scaling exponents: $0.19<\alpha_{1}<1.44$ and $0.018<\alpha_{2}<0.489$. The first index is broadly distributed while the other is peaked at 0.1 .

The application of the first index is justified. In the absence of alpha waves the value of the short-time scaling exponent of the OU Langevin equation is equal to 0.5 , cf. Eq. (6). One of the physiological mechanisms that contribute to the spread of $\alpha_{1}$ are alpha waves which, as discussed in the previous section, enhance short-time growth of diffusion entropy. One can see in Fig. 2 that out of 19 EEG channels the fastest growth of entropy is observed in the occipital channels $\mathrm{O} 1$ and $\mathrm{O} 2$ for which the spectral power of the alpha waves is highest.

The low value of the second index $\alpha_{2}$ and its small standard deviation are not surprising taking into account that the fitting interval, used to determine this index, overlaps the saturation region (cf. Figs. 3 and 4). Note that the asymptotic values of the standard deviation Eq. (7) or diffusion entropy Eq. (14) may be expressed in terms of $D / \lambda$; in other words, they are determined by the interplay of fluctuations and dis-

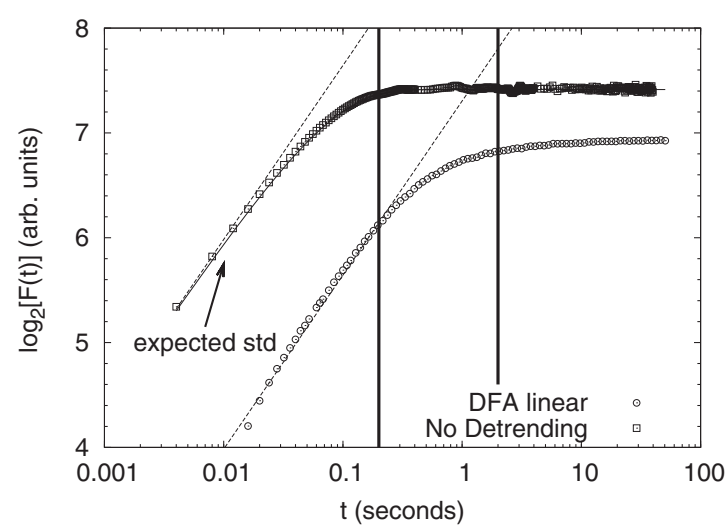

FIG. 5. DFA of the dynamics of the stochastic variable $X(t)$ which is the solution of the OU Langevin equation (1) with $\lambda$ $=0.055$ and $\sigma_{\eta}=40$. The circles correspond to linear detrending. The squares denote the second moment analysis ("zeroth order detrending"). The solid thin line is the analytical expression for the standard deviation Eq. (4). Note that the standard deviation was calculated for the effective dissipation rate $\lambda_{e f f}=\lambda / 2$, see the discussion in the text. The dashed lines were drawn to guide the eyes. Their slopes are equal to 0.5 which is the value of short-time scaling exponent for the OU Langevin equation (1).

sipation. Moreover, monochromatic driving does not affect the saturation level. The driving merely induces periodic oscillations around the asymptotic value Eq. (21). We emphasize that there exists no asymptotic dynamical scaling region for EEG increments as indicated by the fast saturation of diffusion entropy in Fig. 4. The fundamental question arises as to the nature of crossover observed in detrended fluctuation analysis of EEG. Such an effect, reported by Hwa and Ferree, is clearly seen in Fig. 4.

Figure 5 shows detrended fluctuation analysis of the dynamics of the stochastic variable $X(t)$ which is the solution of the OU Langevin equation (1). The circles correspond to linear detrending. The solid thin line is the analytical expression for the standard deviation Eq. (4) calculated for the effective dissipation rate $\lambda_{e f f}=\lambda / 2$ (see discussion of the effective dissipation rate in Sec. III). It turns out that the slow approach of $F(t)$ to the asymptotic value touches the very foundation of DFA algorithm.

DFA has been widely used in neurophysiology because of the nonstationarity of EEG time series. Many argue that detrended fluctuation analysis is particularly applicable to EEG because the drift of EEG baseline, a common artifact, may be interpreted as a semilocal linear trend. DFA adopts this very specific model of nonstationarity Eq. (27) that consists of trends superposed on the signal. In this case trends are the source of nonstationarity. However, it is not true that every nonstationary time series must be of the kind described by Eq. (27). In particular the OU Langevin equation (1) and its extension Eq. (16) do not satisfy the condition Eq. (27). Although one can always arbitrarily decompose the solution of these equations for $X(t)$ into the sum of two terms, these two terms would not have null covariance, which is the underlying assumption of the DFA algorithm. To demonstrate this point in Fig. 5 we plot the result of the second moment analysis of the numerical solution of the OU Langevin model 
(squares). Informally, one may interpret second moment analysis as zeroth order DFA. The two vertical gridlines in this figure were drawn to emphasize that linear detrending delays at least by a decade the approach of $F(t)$ to its asymptotic value. This slow growth may be easily misinterpreted as a pseudoscaling region especially when the DFA is done on very short data segments. Robinson's analysis [8] corroborates our conclusion that the crossover effect observed in DFA of EEG increments is spurious.

\section{CONCLUSIONS}

In a continuing search for clinically significant measures that are superior to those based on simple statistical or spectral analyses one is often forced to relax the stringent assumptions of the relevant mathematical formalisms. This pragmatic approach is fundamentally sound as long as the resulting algorithm does not obscure the underlying character of the physiologic process being investigated.

Fractional Brownian motion (fBm) is the simplest model of fractal time series. The statistics of such a random walk are Gaussian and the autocorrelation function can be long range. The long-range correlations of $\mathrm{fBm}$ and other similar scaling processes may be characterized by a scaling index originally introduced by Hurst to quantify the power-law increase in the second moment with time [12,23]. Detrended fluctuation analysis was developed to detect long-time, power-law scaling of the second moment in the presence of polynomial nonstationarities. Early on, the application of DFA to heart rate variability, namely to the interbeat interval time series, revealed the existence of two scaling regimes [24]. Interestingly enough, the short-time scaling exponent also turned out to be clinically significant. The existence of two scaling regions was later observed in a number of other physiologic phenomena; the dynamics of arterial blood pressure and in cerebral blood flow, to name two $[13,25]$. Over time DFA has become the tool of choice also for analysis of the short-time fluctuations in physiologic time series despite the fact that its validity in this domain had never been demonstrated.

In this work we studied the caveats of fractal time series analysis of EEG time series. In particular, we demonstrated that a short-time scaling exponent can be used to quantify EEG fluctuations. However, in the presence of strong periodic modulation, such as ubiquitous alpha waves, the rationale of such approach is lost since in such circumstances EEG waveforms simply do not scale. We pointed out that the crossover between two scaling regions seen in detrended fluctuation analysis of EEG increments does not originate from the underlying dynamics but is merely an artifact of the algorithm. This artifact is rooted in the failure of the "trend plus signal" paradigm of DFA. Havlin et al. [26] while reviewing the application of statistical physics methods to cardiac diagnostic wrote: "The DFA analysis yields a crossover

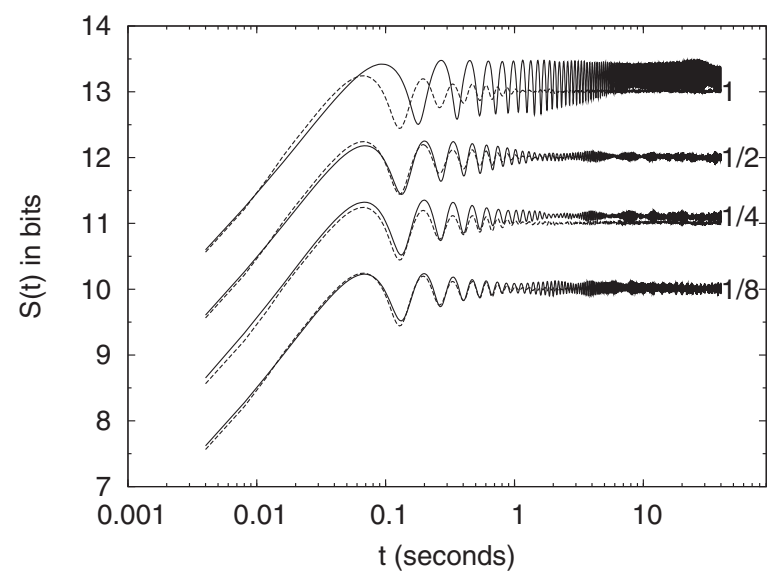

FIG. 6. Diffusion entropy of the EEG increments of channel O1 of Fig. 1) (dashed lines) and the increments of the variable $X(t)$ (solid lines). $X(t)$ is the solution of Eq. (1) with $\lambda=0.055$ and $\sigma_{\eta}$ $=40$, as in Fig. 1, for different values of the stability time $t_{s}$. We consider $t_{s}=1 / 8,1 / 4,1 / 2$, and $1 \mathrm{~s}$. The entropy curves are shifted for clarity.

point for the fractal slope for the scale $m=4$. It should be noted, however, that the crossover point in the DFA analysis is not a sharply defined point, rather the change in fractal slope takes place in a gradual way." The analytical analysis of the OU Langevin model, discussed in this work, has provided the explanation of this effect that may be relevant in interpretation of short-time dynamics of physiological time series.

\section{ACKNOWLEDGMENTS}

M.I. and P.G. thank the Army Research Office for support of this research. The code of the programs used for the EEG analysis (DE and spectrogram) can be downloaded following this link [27]. The code used for the DFA analysis is the one available at PhysioNet website [28]. We thank Andrzej Latka for his comments on the manuscript.

\section{APPENDIX}

Stability time $t_{s}$ is the adjustable parameter of the EEG OU Langevin model Eq. (16). To elucidate the choice of its value, in Fig. 6 we display the diffusion entropy of the increments of four simulated EEGs. The solid curves correspond to $t_{s}=1 / 8,1 / 4,1 / 2$, and $1 \mathrm{~s}$. The other parameters were fixed $\left(\lambda=0.055 \sigma_{\eta}=40\right)$. The curves were vertically shifted to facilitate comparison. The dashed curves, which are in fact identical, denote the diffusion entropy of EEG segment that was used to construct a spectrogram. Recall, that the spectrogram is needed to generate the amplitudes $A_{j}$ and the frequencies $f_{j}$ of the quasiperiodic driving. As we can see a reasonable agreement between the entropy of the simulated and original EEG is achieved for stability time 0.125-0.5 s. 
[1] E. Niedermeyer and F. Da Silva, Electroencephalography: Basic Principles, Clinical Applications, and Related Fields (Lippincott Williams \& Wilkins, New York, 2004).

[2] K. Linkenkaer-Hansen, V. Nikouline, J. Palva, and R. Ilmoniemi, J. Neurosci. 21, 1370 (2001).

[3] J. Lee, D. Kim, I. Kim, K. Park, and S. Kim, Comput. Biol. Med. 32, 37 (2002).

[4] R. C. Hwa and T. C. Ferree, Phys. Rev. E 66, 021901 (2002).

[5] J. Yuan, B. Zheng, C. Pan, Y. Wu, and S. Trimper, Physica A 364, 315 (2006).

[6] S. M. Cai, Z. H. Jiang, T. Zhou, P. L. Zhou, H. J. Yang, and B. H. Wang, Phys. Rev. E 76, 061903 (2007).

[7] C.-K. Peng, S. V. Buldyrev, S. Havlin, M. Simons, H. E. Stanley, and A. L. Goldberger, Phys. Rev. E 49, 1685 (1994).

[8] P. A. Robinson, Phys. Rev. E 67, 032902 (2003).

[9] N. Scafetta, P. Hamilton, and P. Grigolini, Fractals 9, 193 (2001).

[10] M. Ignaccolo, M. Latka, W. Jernajczyk, P. Grigolini, and B. West, J. Biol. Phys. 36, 185 (2010).

[11] K. Lindenberg and B. West, The Nonequilibrium Statistical Mechanics of Open and Closed Systems (VCH, New York, 1990).

[12] H. Hastings, G. Sugihara, H. Hartings, and H. Hastings, Fractals-A User's Guide for the Natural Sciences (Oxford University Press, Oxford, 2002).

[13] B. West, Where Medicine Went Wrong: Rediscovering the Path to Complexity (World Scientific, Singapore, 2006).
[14] C. Shannon, Bell Syst. Tech. J. 27, 379 (1948).

[15] N. Wiener, Cybernetics (MIT Press, Cambridge, 1950).

[16] P. Allegrini, J. Bellazzini, G. Bramanti, M. Ignaccolo, P. Grigolini, and J. Yang, Phys. Rev. E 66, 015101(R) (2002).

[17] M. Ignaccolo, P. Allegrini, P. Grigolini, P. Hamilton, and B. West, Physica A 336, 623 (2004).

[18] P. Grigolini, D. Leddon, and N. Scafetta, Phys. Rev. E 65, 046203 (2002).

[19] N. Scafetta and B. J. West, Phys. Rev. Lett. 90, 248701 (2003).

[20] A. L. Goldberger, L. A. Amaral, L. Glass, J. M. Hausdorff, P. C. Ivanov, R. G. Mark, J. E. Mietus, G. B. Moody, C. K. Peng, and H. E. Stanley, Circulation 101, E215 (2000).

[21] A. Bashan, R. Bartsch, J. Kantelhardt, and S. Havlin, Physica A 387, 5080 (2008).

[22] M. Ignaccolo, M. Latka, W. Jernajczyk, P. Grigolini, and B. West, Phys. Rev. Lett. e-print arXiv:1001.4103.

[23] J. Feder, Fractals (Plenum, New York, 1988).

[24] C.-K. Peng, S. Havlin, H. E. Stanley, and A. L. Goldberger, Chaos 5, 82 (1995).

[25] M. Latka, M. Glaubic-Latka, D. Latka, and B. West, Chaos, Solitons Fractals 20, 165 (2004).

[26] S. Havlin, L. A. Amaral, Y. Ashkenazy, A. L. Goldberger, P. Ch. Ivanov, C. K. Peng, and H. E. Stanley, Physica A 274, 99 (1999).

[27] http://www.duke.edu/ mi8/software.html

[28] http://www.physionet.org/physiotools/dfa 